\title{
Prevalence and associated factors of metabolic syndrome in adults: a population-based epidemiological survey in Jiangxi province, China
}

\author{
Li Ting $\mathrm{Wu}^{1,2+} \mathbb{D}$, Yun Feng Shen ${ }^{1,3+}$, Lei Hu ${ }^{1}$, Mei Ying Zhang ${ }^{1}$ and Xiao Yang Lai ${ }^{1,3^{*}}$
}

\begin{abstract}
Background: Metabolic syndrome (MS) has abruptly increased in China in the past two decades, gradually representing an important public health threat over the years. Here, we firstly reported the prevalence and associated factors of metabolic syndrome in Jiangxi province, China.

Methods: A population-based cross-sectional survey was performed in Jiangxi province, China, from April to August 2015. MS was diagnosed by International Diabetes Federation (IDF) and Chinese Diabetes Society (CDS) criteria, respectively. Factors associated with MS were investigated by multivariate logistic regression.

Results: A total of 2665 residents aged over 18 years were enrolled, and 2580 effectively participated. According to IDF and CDS criteria, age-standardized prevalence of MS were 21.1 and 15.2\% in all participants, respectively; prevalence were $19.6 \%$ or $17.1 \%$ in men, and $22.7 \%$ or $13.0 \%$ in women, based on these respective criteria. Rural participants had a significantly higher prevalence than urban individuals, so did rural females. Prevalence in males did not differ between rural and urban participants. Factors independently associated with MS were low education level and menopausal state.

Conclusions: To the best of our knowledge, this was the latest study on MS prevalence in Jiangxi province. In conclusion, MS prevalence is high in Jiangxi province. Considering the unhealthy lifestyle, education is urgently needed to prevent the rapid increase of MS prevalence.
\end{abstract}

Keywords: Metabolic syndrome, Prevalence, Associated factors

\section{Background}

Metabolic syndrome (MS) is a constellation of interrelated metabolic disturbances based on insulin resistance, including visceral adiposity, atherogenic dyslipidemia, elevations of blood pressure (BP) and glucose, endothelial dysfunction, prothrombotic and proinflammatory states; it is associated with greatly increased morbidity and mortality of cardiovascular disease (CVD) and type 2

\footnotetext{
* Correspondence: 1640852510@qq.com

${ }^{\dagger}$ Li Ting Wu and Yun Feng Shen contributed equally to this work. ${ }^{1}$ Department of Endocrinology and Metabolism, The Second Affiliated Hospital of Nanchang University, No.1, Minde Road, Donghu District, Nanchang 330006, China

${ }^{3}$ Institute for the Study of Endocrinology and Metabolism Diseases in Jiangxi Province, No.1, Minde Road, Donghu District, Nanchang 330006, China Full list of author information is available at the end of the article
}

diabetes mellitus (T2DM) [1-5]. In the global context of urbanization and the spread of unhealthy life-style, the high prevalence of MS gradually over-burdens public health [6].

Interestingly, different MS prevalence have been presented in published studies assessing the Chinese population [7-17]. MS prevalence was reported at $13.3 \%$ in 2001, rapidly rising to $18.2 \%$ in 2009 in China Health and Nutrition Survey, according to the Chinese Diabetes Society (CDS) criteria [7, 14]. Paradoxically, a prevalence of $9.82 \%$ found in 2014 was lower than that of 2009 by the same criteria [15]. To the best of our knowledge, at least three reasons may explain these results. Firstly, diverse dietary habits in different regions could represent an important cause of the disparate prevalence of MS.

(c) The Author(s). 2020 Open Access This article is distributed under the terms of the Creative Commons Attribution 4.0 International License (http://creativecommons.org/licenses/by/4.0/), which permits unrestricted use, distribution, and reproduction in any medium, provided you give appropriate credit to the original author(s) and the source, provide a link to the Creative Commons license, and indicate if changes were made. The Creative Commons Public Domain Dedication waiver (http://creativecommons.org/publicdomain/zero/1.0/) applies to the data made available in this article, unless otherwise stated. 
The MS prevalence in Jilin province and the Yan-an region of Shanxi province were 22.4 and $26.4 \%$ according to International Diabetes Federation (IDF) criteria, respectively $[13,18]$. Secondly, different diagnosis criteria also could contribute to these differences. As shown previously, MS prevalence was $12.6 \%$ by the CDS criteria in the Hangzhou region of Zhejiang province, and $7.3 \%$ by IDF criteria in Guangdong province [11, 12]. Thirdly, different socioeconomic status (SES) might be a vital factor leading to the differences in MS prevalence. As reported previously, MS prevalence was $33.9 \%$ by modified IDF criteria in affluent urban districts in Shanghai, and $11.8 \%$ by IDF criteria in un-developed rural regions of NingXia province [16, 17].

Both IDF and CDS criteria for MS are two widely used criteria for clinical and research purposes [9, 14]. Most components of MS are similar between IDF and CDS criteria, although some slight differences remain. IDF criteria emphasize on central obesity evaluated by waist circumstance (WC), while CDS criteria stressed on overall body obesity evaluated by body mass index (BMI). Besides, IDF criteria consider central obesity as prerequisite, and have stricter cut-offs for blood pressure and blood glucose levels.

Jiangxi province is an under-developed province in central China, with 45 million Han Chinese or so. With sustained economic growth, the Western lifestyle in local populations has been developed in the past two decades. Unfortunately, although many studies of MS have been performed in other regions of China, little information is available for Jiangxi province. In the present study, we investigated MS prevalence based on the IDF and CDS criteria in Jiangxi province, in a cross-sectional survey sponsored by the TIDE (Thyroid disorders, Iodine status and Diabetes: a national Epidemiological survey) study group.

In terms of the associated factors of MS, education level, gender, residential region and income are proposed to be related with MS [19]. But these factors may be correlated with different SES or criteria used for defining MS [20]. Moreover, they might have region-specificity [21]. Therefore, the current study subsequently determined the independently associated factors for MS in Jiangxi province.

\section{Methods}

\section{Study design and population}

The current data originated from the epidemiological data collected by the TIDE. This population-based, cross-sectional, epidemiological survey was performed in Jiangxi province from April to August in 2015. Sample size was estimated to meet generally recommended requirements for precision in a random sampling survey. Assuming the confidence interval was 95\% and the MS prevalence $10 \%$. The permissible relative sampling error was $4 \%$. Therefore, the total sample size was 217 . In this study, 2665 adults were invited and 2580 effectively attended, giving a response rate of $96.8 \%$. The sample size achieved the minimum requirement for the sampling method.

A cluster sampling method was applied in this study (Fig. 1). (1) According to the economical size of each city and county in Jiangxi province provided by Statistical Bureau, areas which fell in the 25th to 75th percentiles of the Gross Domestic Product (GDP) level were included. Besides, considering the data accessibility, sparsely inhabited districts with higher or lower economical sizes were excluded. (2) In the above areas, one urban city (Nanchang) and one rural county (Dexing) were chosen using a simple random sampling method. Afterwards, one community (Qingyunpu) and four villages (Diaozhong, Yincheng, Raoer and Huangbai) were picked using the above method. (3) The participants were restricted to local residents aged over 18 years old who had been living at the study sites for at least 5 years. Pregnant women were excluded. According to residential registration, all of the eligible candidates were included.

\section{Questionnaire, anthropometric measurements and blood sample investigation}

Firstly, individual demographic characteristics and disease history questionnaire were completed. Demographic and clinical information included age, sex, level of education, marital status, menstrual condition, family annual income, occupational status, smoking, history of diseases such as diabetes, hypertension, dyslipidemia and hyperuricemia, medications for diseases and family history of diabetes. In multiple logistic regression analysis, age was classified into 3 groups, including $\leq 40,41-65$ and $\geq 66$ years old. Education was classified into 3 levels, including primary school and below, middle and high school, and college and above. Family annual income was graded into 4 levels: (1)<30,000; (2) 30,000 50,000; (3) 50,000 100,000; (4)>100,000 Chinese yuan (100 $\mathrm{CNY}=14.56 \mathrm{USD})$. Occupational status was categorized into student, worker (farmer), house worker (retired), clerk and others. Smoking was defined as at least 100 cigarettes consumed prior to the survey and was further divided into two groups on the basis of the frequency of cigarettes consumption $(<1$ cigarette per day or $\geq 1$ cigarette per day). Amenorrhea was defined as not having a period for at least 6 months or 3 menstrual cycles.

Anthropometric data, including BP, heart rate (HR), WC, height and body weight, were measured by recommended standard procedures. In brief, BP and HR were average values of two separate measurements taken at 5min intervals. Weight and height were measured without shoes or heavy garments. BMI $\left(\mathrm{kg} / \mathrm{m}^{2}\right)$ was determined 


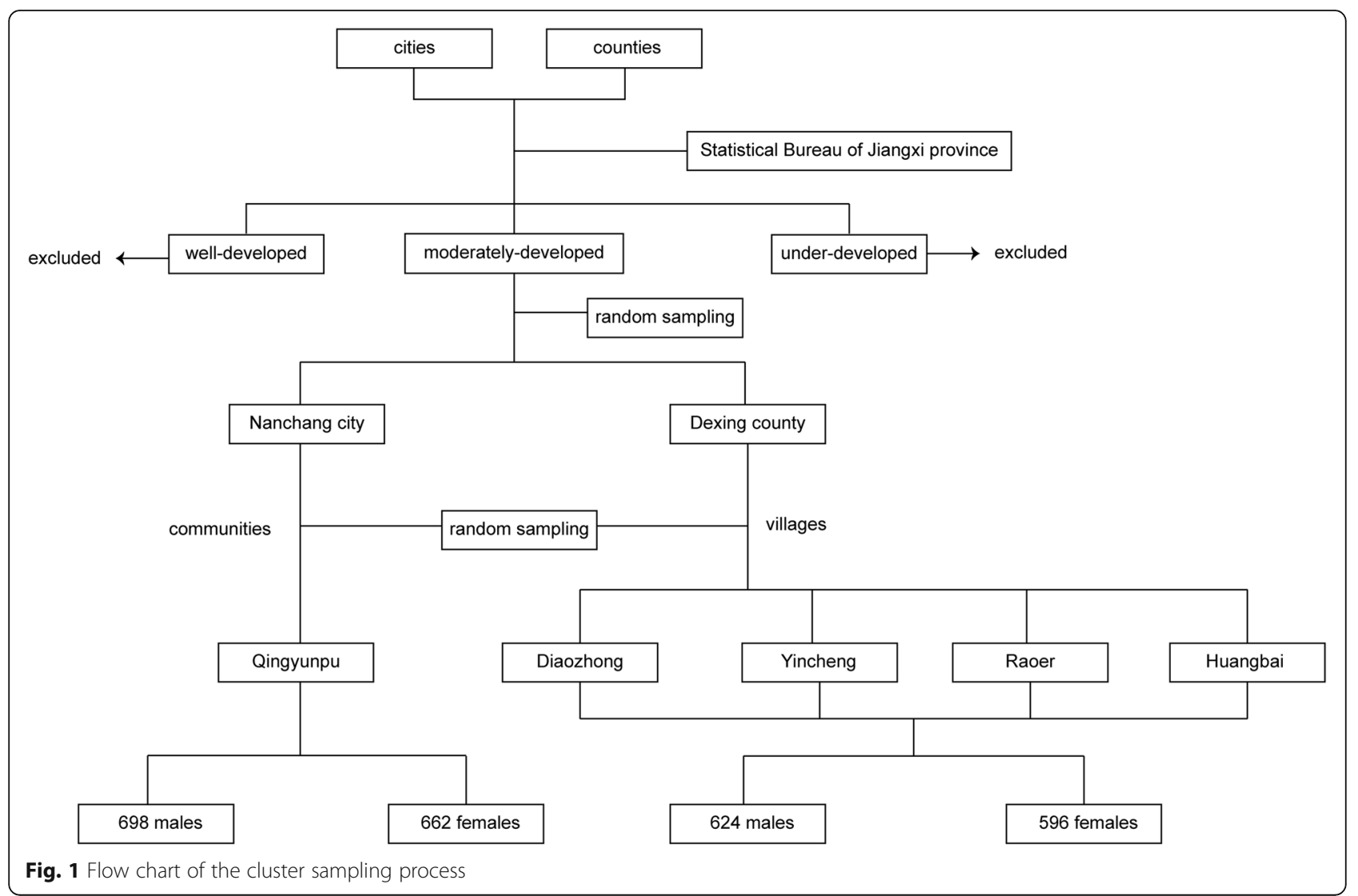

by dividing the weight $(\mathrm{kg})$ by height $(\mathrm{m})$ squared. WC was measured in the erect position at the middle of the lowest rib and the superior border of the iliac crest.

Blood samples were obtained after at least $10 \mathrm{~h}$ of fasting to determine fasting blood chemistry parameters. Afterward, all subjects were given a standard $2 \mathrm{~h}-75 \mathrm{~g}$ oral glucose tolerance test (OGTT). All serum parameters were detected on a Mindray (Mindray Medical International Limited, China) automatic biochemistry analyzer. Serum total cholesterol (TC) and triglyceride (TG) levels were determined by enzymatic methods. Serum low density lipoprotein cholesterol (LDL-C) and high density lipoprotein cholesterol (HDL-C) amounts were measured by the direct method. Serum uric acid (UA) content was measured by the uric acid enzymeperoxide enzyme coupling method. Fasting plasma glucose (FPG) and OGTT-2 h plasma glucose (2 h PG) were determined by the glucose oxidase method. Glycosylated hemoglobin A1c (HbA1c) was measured by high pressure liquid chromatography. All the procedures were executed by experienced laboratory technicians.

\section{Diagnosis of metabolic syndrome}

MS was diagnosed based on IDF or CDS criteria (Chinese specific) [9, 14]. These two criteria are described in Additional file 1: Table S1.
Data collection and statistical analyses

In this study, data collection was performed by trained medical professionals at the Second Affiliated Hospital of Nanchang University. Data analysis was limited to individuals who had completed all procedures, comprising 1322 (51.2\%) males and 1258 (48.8\%) females; 1360 (52.7\%) participants lived in urban areas, and 1220 (47.3\%) in rural regions.

An EpiData (EpiData Association, Odese, Denmark) database was established, and all data were analyzed by SPSS (Statistical Program for Social Sciences, version 20.0). Continuous variables were described as mean (standard error, SE) and analyzed by student's $t$-test. Categorical variables are presented as number and percentage, and analyzed by the Chi-square test. The official 2010 census data of China was used to determine agestandardized ratios.

We analysis the associated factors for MS with logistic regression models. We reported the odd ration (OR) with $95 \%$ CI. Covariates included in the multivariable logistic regression models were profession (student [reference] vs worker vs clerk vs houseworker vs others), family annual income (below 30,000 [reference] vs 30, $000-50,000$ vs $50,000-100,000$ vs above 100,000 ), age (18-40 [reference] vs 41-65 vs above 66), education level (primary school or below [reference] vs middle or 

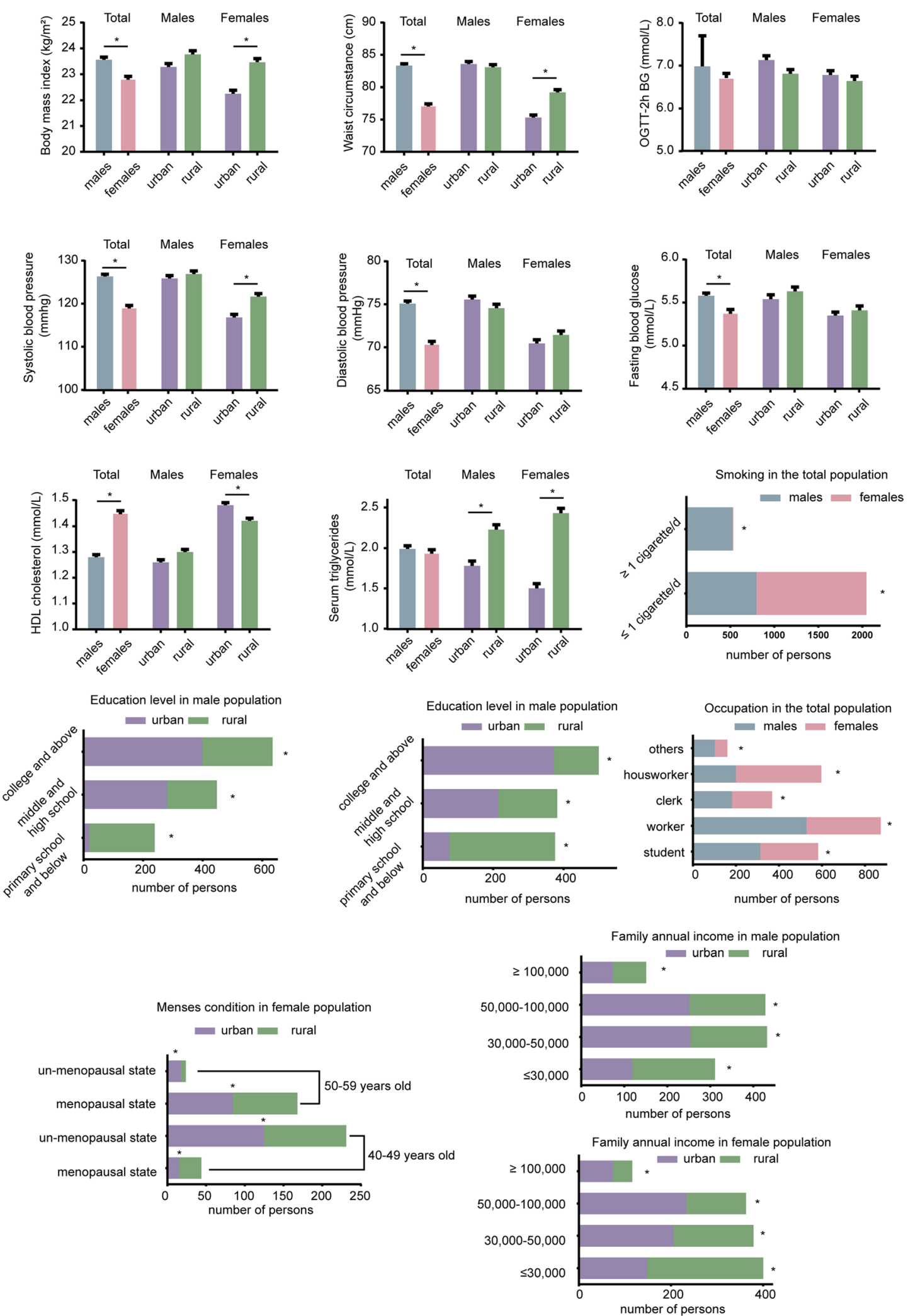

Fig. 2 Characteristics of the survey population. ${ }^{*} p<0.05$ for men vs women; or urban vs rural as indicated in the figure. HDL cholesterol, high density lipoprotein cholesterol; OGTT-2 h BG, blood glucose of $2 \mathrm{~h}$ oral glucose tolerance test 
high school vs college or above), family history of diabetes (yes [reference] vs no), smoke (no or less than 1 cigarette/d [reference] vs more than 1 cigarette/d), Menses condition (menopausal state [reference] vs unmenopausal state). We also investigated whether the associated factors for MS varied by region and gender by stratified multivariable analyses (in urban males, urban females, rural males and rural females respectively).

\section{Results}

\section{Characteristics of the survey population}

A total of 1322 adult males and 1258 adult females were finally included and evaluated in this cross-sectional study. The characteristics of these individuals are shown in Fig. 2. Though no significant differences were found in TG levels between men and women, rural participants showed significantly higher levels than urban counterparts. Men had higher BMI, WC, SBP and DBP, but HDL-C levels were higher in women. No significant differences were seen in BMI, WC and SBP between urban and rural men. In contrast, rural women had greater levels of the above anthropometric values than urban women. For HDL-C, rural women showed significantly lower levels. Besides, urban women had delayed menopause compared with their rural counterparts. In urban areas, participants had higher education and income levels. The smoking rate was nearly $40 \%$ in men and was significantly higher than that of women. Meanwhile, more women performed housework than men.

\section{MS prevalence based on different criteria}

The prevalence of MS are displayed in Table 1. Of the 2580 subjects, 542 (21.0\%) were diagnosed with MS based on IDF criteria. The age-adjusted prevalence was $21.1 \%$ for the whole population, with 22.6 and $19.6 \%$ in females and males, respectively. Significant differences were observed between rural (23.9\%) and urban (18.5\%) areas, especially in female population (Fig. 4). With population aging, MS prevalence increased (Fig. 3). No significant gender differences were observed in total prevalence, but women had higher prevalence in elderly groups than men $(47.9 \%$ vs 27.6 and $41.1 \%$ vs $20.4 \%$, in 60-69 and $>70$ years, respectively, $p<0.01$ ). In perimenopausal women groups, MS prevalence in rural areas were significantly higher compared with those of urban regions ( $32.6 \%$ vs 14.3 and $47.8 \%$ vs $28.4 \%$, in 40 49 and $50-59$ years, respectively, $p<0.01$ ). Besides, such difference was also found in $18-29$ years females $(7.3 \%$ vs $1.1 \%$, respectively, $p<0.01$ ).

Of the 2580 subjects, 390 (15.1\%) were diagnosed with MS by CDS criteria. The age-adjusted prevalence was $15.2 \%$ for the whole population, including $17.1 \%$ in men and $13.0 \%$ in women. Similarly, there was a significant age-related increase in MS prevalence (Fig. 3). MS
Table 1 Age- and sex-specific prevalence (\%) of MS based on different definitions

\begin{tabular}{|c|c|c|c|c|c|c|}
\hline & Men & IDF & Total & Men & CDS & Total \\
\hline & & \multicolumn{3}{|l|}{ Women } & \multicolumn{2}{|l|}{ Women } \\
\hline \multicolumn{7}{|l|}{ Urban } \\
\hline $18-29$ & 3.4 & $1.1^{* *}$ & $2.3^{* *}$ & 1.1 & 0 & 0.6 \\
\hline $30-39$ & 19.9 & 15.6 & 17.8 & 12.8 & 8.9 & 10.9 \\
\hline $40-49$ & 22.7 & $14.3^{* *}$ & $18.7 * *$ & $19.5^{*}$ & $6.4^{* *}$ & 13.3 \\
\hline $50-59$ & 26.4 & $28.4^{* *}$ & $27.4^{* *}$ & 24.5 & $14.7^{* *}$ & $19.7^{* *}$ \\
\hline $60-69$ & 30.9 & 46.2 & 38.4 & 32.4 & 40.0 & 36.1 \\
\hline$\geq 70$ & 26.0 & 38.6 & 31.9 & 30.0 & 20.5 & 25.5 \\
\hline Total, crude & 18.8 & $18.0 * *$ & $18.5^{* *}$ & $16.2 *$ & $10.7^{* *}$ & $13.5^{* *}$ \\
\hline \multicolumn{7}{|l|}{ Rural } \\
\hline $18-29$ & 5.5 & 7.3 & 6.4 & 1.8 & 0.7 & 1.3 \\
\hline $30-39$ & 22.0 & 16.7 & 19.3 & 15.4 * & 6.7 & 11.1 \\
\hline $40-49$ & 27.9 & 32.6 & 30.2 & 22.9 & 14.1 & 18.5 \\
\hline $50-59$ & 33.7 & 47.8 & 40.5 & 30.5 & 28.9 & 29.7 \\
\hline $60-69$ & $23.7^{*}$ & 50.0 & 36.3 & 30.5 & 37.0 & 33.6 \\
\hline$\geq 70$ & $14.0 *$ & 43.5 & 29.2 & 27.9 & 41.3 & 34.8 \\
\hline Total, crude & $20.4^{*}$ & 27.7 & 23.9 & 18.1 & 15.6 & 16.9 \\
\hline \multicolumn{7}{|l|}{ total population } \\
\hline $18-29$ & 4.4 & 4.0 & 4.2 & 1.5 & 0.3 & 0.9 \\
\hline $30-39$ & 20.8 & 16.1 & 18.5 & $14.0 *$ & 7.8 & 11.0 \\
\hline $40-49$ & 25.2 & 23.3 & 24.3 & $21.1 *$ & 10.2 & 15.8 \\
\hline $50-59$ & 29.9 & 37.5 & 33.6 & 27.4 & 21.4 & 24.4 \\
\hline 60-69 & $27.6 *$ & 47.9 & 37.4 & 31.5 & 38.7 & 35.0 \\
\hline$\geq 70$ & $20.4^{*}$ & 41.1 & 30.6 & 29.0 & 31.1 & 30.1 \\
\hline Age-standardized & 19.6 & 22.7 & 21.1 & $17.1^{*}$ & 13.0 & 15.2 \\
\hline
\end{tabular}

prevalence was significantly higher in men compared with women $(p<0.01)$, especially in the $30-39(14.0 \%$ vs $7.8 \%, p<0.05)$ and $40-49(21.1 \%$ vs $10.2 \%, p<0.05)$ years age groups. In peri-menopausal women, MS prevalence in rural areas were significantly higher than those of urban regions $(14.1 \%$ vs 6.4 and $28.9 \%$ vs $14.7 \%$ in the 40-49 and 50-59 years age groups, respectively, $p<0.05)$. Similar to IDF criteria, rural participants showed significantly higher prevalence than urban counterparts. Besides, rural females had significantly higher prevalence (Fig. 4).

\section{Independent associated factors for MS}

Overall, the multivariate adjusted OR for MS in CDS criteria continually decreased with increasing education level, from $0.851(0.645-1.123)$ in the middle or high school group to $0.670(0.512-0.877)$ in the college and above group. Unfortunately, we haven't identified any of associated factors for MS by IDF 

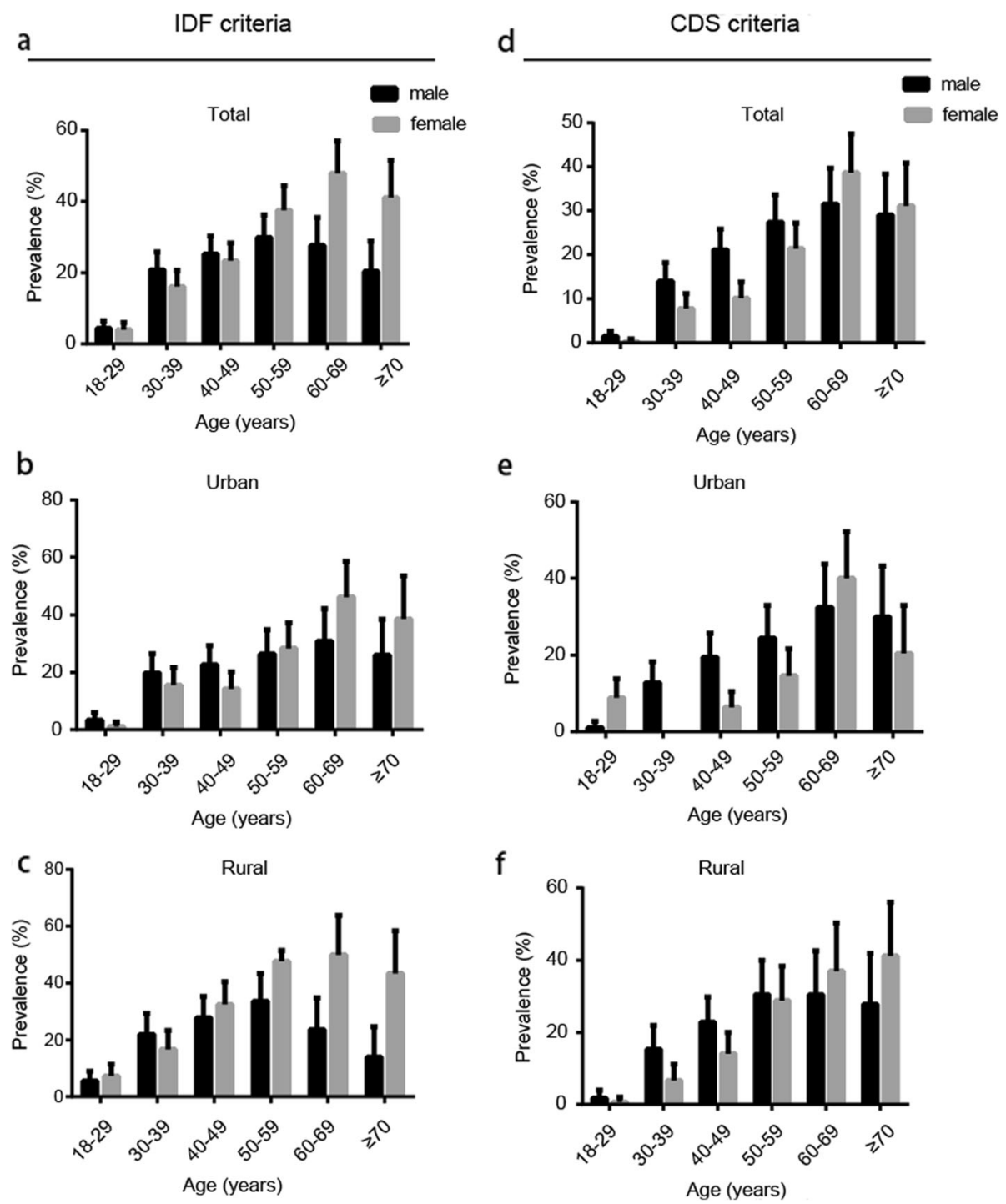

Fig. 3 Age-specific and age-standardized prevalence of MS in Jiangxi Province in 2015, based on IDF (a-c) and CDS (d-f) criteria, respectively. Error bars indicate $95 \%$ confidence intervals

criteria. Hence, we performed further analysis based on CDS criteria. In stratified logistic regression analysis, we found that the risk of developing MS is $69 \%$ less in middle or high school group and $76 \%$ less in college or above group than primary school or below group (reference) in men from urban regions. Higher education level did not protect women from MS in urban areas. Compared with nonmenopausal women, menopausal females had a higher risk of developing MS, by about 1.5-fold for urban females. Occupation, living area, elderly age, family annual income, smoking and family history of T2DM were not independently associated with MS in either gender (Table 2).

\section{Prevalence of individual metabolic abnormalities}

Using the IDF definition of MS, high TG level was the most frequent element in both genders. The prevalence of high blood pressure was two times higher in men than in women (Table 3). Using CDS definition of MS, high blood glucose level was the most frequent, while high HDL cholesterol level the least (Table 4).

\section{Discussion}

In this population-based, cross-sectional, epidemiological survey, we found that overall age-adjusted prevalence of MS were 21.1 and $15.2 \%$, according to IDF and CDS criteria, respectively. MS prevalence gradually increased with age. Rural individuals, especially females, had 


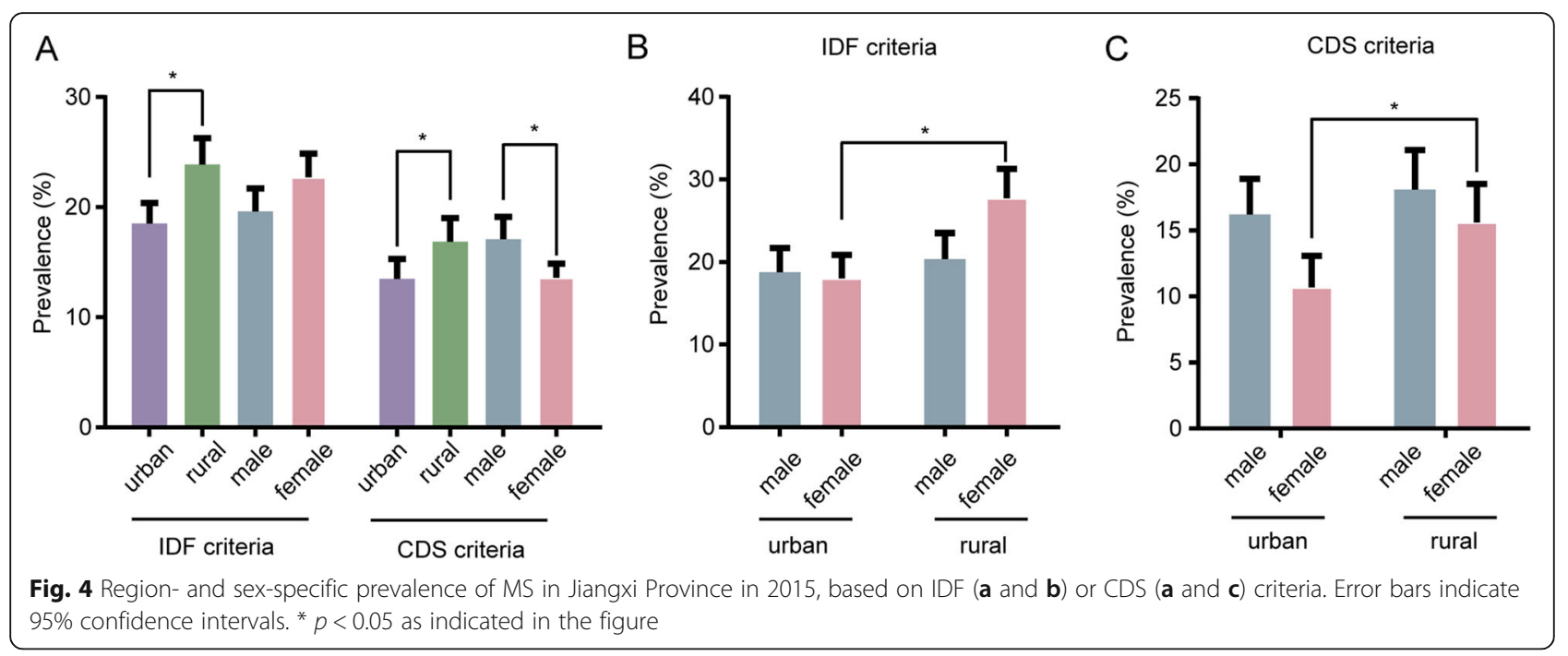

higher prevalence of MS, irrespective of the diagnostic criteria used (IDF or CDS criteria). However, different diagnostic criteria revealed opposite outcomes regarding sex prevalence. Moreover, low education level and the menopausal state could be independent associated factors for MS.

MS increases the risk of T2DM, CVD and cancer in the general population, resulting in an enormous economic burden for the society $[1,5]$. MS prevalence were 19.85 and $9.95 \%$ in 2013 in Jiangxi province based on IDF and CDS criteria, respectively, as reported by Cheng et al. [22].. After 2 years, a significant increase was observed in this survey. This terrible phenomenon suggests that effective prevention measures should be taken immediately.

A higher prevalence of MS for the elderly has been observed in worldwide surveys $[6,12,18,23,24]$. With increased population aging in China, MS-related economic burden would challenge medical workers and public health. In this study, MS prevalence increased with age and reached a peak in 60-69 years old individuals diagnosed by both criteria. However, according to IDF criteria, the male prevalence rate peaked at $50-59$ years old. These trends were also reported by Li et al. [13] in the Yan-an region. In contrast, peak ages were reduced than those of Chen's study in Shanghai [25]. For

Table 2 Independent associated factors of MS using multiple logistic regression

\begin{tabular}{|c|c|c|c|}
\hline \multicolumn{2}{|l|}{ Participants } & \multicolumn{2}{|c|}{ 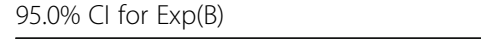 } \\
\hline Factors & $\mathrm{OR} / \operatorname{Exp}(\mathrm{B})$ & Lower & Upper \\
\hline \multicolumn{4}{|l|}{ All participants } \\
\hline \multicolumn{4}{|l|}{ Education } \\
\hline Primary school or below (reference) & 1.0 & 1.0 & \\
\hline Middle or high school & 0.851 & 0.645 & 1.123 \\
\hline College or above & 0.670 & 0.512 & 0.877 \\
\hline \multicolumn{4}{|l|}{ Urban males } \\
\hline \multicolumn{4}{|l|}{ Education } \\
\hline Primary school or below (reference) & 1.0 & 1.0 & \\
\hline Middle or high school & 0.310 & 0.116 & 0.823 \\
\hline College or above & 0.237 & 0.090 & 0.624 \\
\hline \multicolumn{4}{|l|}{ Urban females } \\
\hline \multicolumn{4}{|l|}{ Menses condition } \\
\hline menopausal state (reference) & 1.0 & 1.0 & \\
\hline un-menopausal state & 0.645 & 0.422 & 0.984 \\
\hline
\end{tabular}


Table 3 Sex-specific prevalence (\%) of individual metabolic abnormalities based on IDF definition

\begin{tabular}{|c|c|c|c|c|c|c|}
\hline \multirow[t]{3}{*}{ Metabolic abnormality } & \multicolumn{6}{|c|}{ Prevalence } \\
\hline & \multicolumn{2}{|c|}{ Men $(N=258)$} & \multicolumn{2}{|c|}{ Women $(N=164)$} & \multicolumn{2}{|c|}{ Total $(N=390)$} \\
\hline & $\%$ & $95 \% \mathrm{Cl}$ & $\%$ & $95 \% \mathrm{Cl}$ & $\%$ & $95 \% \mathrm{Cl}$ \\
\hline Obesity & 34.1 & $28.3-39.9$ & 37.7 & $32.0-43.3$ & 34.1 & $30.1-38.1$ \\
\hline High serum TG level & 49.6 & $43.5-55.8$ & 41.5 & $35.8-47.3$ & 45.4 & $41.2-49.6$ \\
\hline High HDL cholesterol & 19.8 & $14.9-24.7$ & 32.0 & $26.6-37.5$ & 26.2 & $22.5-29.9$ \\
\hline High blood pressure & 45.0 & $38.9-51.1$ & 22.2 & $17.3-27.0$ & 33.0 & $29.1-37.0$ \\
\hline High serum glucose level & 31.0 & $25.3-36.7$ & 23.9 & $19.0-28.9$ & 27.3 & $23.5-31.1$ \\
\hline
\end{tabular}

preventing morbidity and mortality of MS related diseases, more attention should be focused on individuals of 50-69 years old in Jiangxi province.

A difference in MS prevalence may exist due to diverse living areas from north to south China. Though close crude prevalence were shown between our results and 2013 data in the Chinese population, a higher MS prevalence was reported in northern China, for example in the Yan-an and Jilin regions $[10,13,26]$. Besides, a more rapid economic development compared with the national average in Jiangxi in recent years has contributed to the MS prevalence rising to near that of Shanghai [25]. Worldwide, diverse races and diet cultures might be associated with MS prevalence. Based on the IDF definition, MS prevalence were reported to be $31.4 \%$ in India, $27.5 \%$ in Malaysia, and $32.8 \%$ in Mongolia, which were higher than the corresponding values in China and Jiangxi province [18, 23, 27].

More participants were inclined to be diagnosed with MS by the IDF criteria. Both diagnostic criteria have different cutoffs for BP, TC and glucose levels. Besides, IDF criteria rely on WC to define central obesity as a preliminary condition for diagnosing MS, while CDS criteria are based on BMI. This discrepancy might also lead to the opposite outcome of prevalence observed between genders. In this study, we found that about $20 \%$ women had low BMI $\left(<25 \mathrm{~kg} / \mathrm{m}^{2}\right)$ with over $80 \mathrm{~cm} \mathrm{WC}$, while $8 \%$ men had low BMI $\left(<25 \mathrm{~kg} / \mathrm{m}^{2}\right)$ with over $90 \mathrm{~cm}$ WC. On the other hand, over $23 \%$ men had higher BMI $\left(>25 \mathrm{~kg} / \mathrm{m}^{2}\right)$ with smaller WC $(<90 \mathrm{~cm})$, and $7 \%$ women had higher BMI $\left(>25 \mathrm{~kg} / \mathrm{m}^{2}\right)$ with smaller
WC $(<80 \mathrm{~cm})$. In other words, females had higher WC with lower BMI, while males had higher BMI with lower WC. Similar results were reported in the Chinese population between 2010 and 2013, but not in Gu's study performed in 2005 [8, 15, 26]. This suggests that sex-specific criteria need to be considered in diagnosing MS.

The associated factors for MS were evaluated by multiple logistic regression analysis in this study. Low education and the menopausal state were independent associated factors for adults in Jiangxi province. As shown above, individuals with low education had a higher risk of developing MS diagnosed by CDS criteria. We assumed that urban residents had higher odds of getting education resources, and received more information about balanced diet. As a tradition, the diet habit in Jiangxi province is fatty and salty. Blood TG levels, closely related to diet, were higher in rural individuals, suggesting the traditional diet habit is more common in rural areas of Jiangxi province. Therefore, a higher prevalence of MS in rural areas was observed in this study, regardless of the diagnostic criteria. Hence, a healthy diet should be recommended in rural areas of Jiangxi province. Similar to a previous study, a significant negative association of non-menopausal state with MS prevalence was shown in women [28]. This association is likely mediated by the testosterone/estradiol ratio. However, when compared with other regions in the world, region-specific factors may exist, such as alcohol

Table 4 Sex-specific prevalence (\%) of individual metabolic abnormalities based on CDS definition

\begin{tabular}{|c|c|c|c|c|c|c|}
\hline \multirow[t]{3}{*}{ Metabolic abnormality } & \multicolumn{6}{|c|}{ Prevalence } \\
\hline & \multicolumn{2}{|c|}{ Men $(N=258)$} & \multicolumn{2}{|c|}{ Women $(N=164)$} & \multicolumn{2}{|c|}{ Total $(N=390)$} \\
\hline & $\%$ & $95 \% \mathrm{Cl}$ & $\%$ & $95 \% \mathrm{Cl}$ & $\%$ & $95 \% \mathrm{Cl}$ \\
\hline Obesity & 33.6 & $27.4-39.8$ & 31.7 & $24.5-38.9$ & 32.8 & $28.1-37.5$ \\
\hline High serum TG level & 39.4 & $33.0-45.8$ & 55.5 & $47.8-63.2$ & 46.2 & $41.2-51.1$ \\
\hline High HDL cholesterol & 7.1 & $3.7-10.4$ & 6.1 & $2.4-9.8$ & 6.7 & $4.2-9.2$ \\
\hline High blood pressure & 23.0 & $17.5-28.5$ & 24.4 & $17.7-31.0$ & 23.6 & $19.4-27.8$ \\
\hline High serum glucose level & 42.0 & $35.6-48.5$ & 67.1 & $59.8-74.3$ & 52.6 & $47.6-57.5$ \\
\hline
\end{tabular}


consumption had a major association with MS in Mongolian population [18]. Therefore, region-specific measurement was needed to prevent the increase of MS prevalence.

The definition of MS remains controversial. It was reported that IDF and ATP III criteria have good consistency [24]. Here, we compared two other systems, including IDF and CDS criteria. It is well-known that China is a large population country, with the geography, climate, living conditions and diet varying from south to north. Identical MS criteria may not be suitable for different people. To the best of our knowledge, IDF criteria are more suitable to northwest Chinese individuals who have elevated WC compared with the others [13]. Besides, different criteria have distinct predictive values in prognosis. The IDF criteria have a reduced value for predicting all-cause mortality in T2DM patients, though it is better than ATP-III criteria [29].

This study had several strengths. Firstly, rigorous training was performed for all study staff before survey initiation. Secondly, detailed information about medications for metabolic disorders were collected. In several previous national or regional studies reporting high MS prevalence in the Chinese population, medications for lipid-, glucose- or blood pressure-lowering were not documented [14]. Therefore, the actual prevalence of MS could have been underestimated. As an epidemiological survey, this study also had limitations. Since only one urban community and four rural villages were included, the sample was small and only partially representative. Besides, our study did not investigate physical activity and dietary habit, which may miss some important information. Furthermore, the occurrence of negative outcome correlated with MS was not predicted, and further investigation is required for clarification.

\section{Conclusions}

To the best of our knowledge, this was the latest study on MS prevalence in Jiangxi province. In conclusion, MS prevalence is high in Jiangxi province. Considering the unhealthy lifestyle, education is urgently needed to prevent the rapid increase of MS prevalence.

\section{Supplementary information}

Supplementary information accompanies this paper at https://doi.org/10. 1186/s12889-020-8207-x.

Additional file 1: Table S1. Criteria of metabolic syndrome diagnosis in the IDF and CDS systems.

\section{Abbreviations}

BMI: Body Mass Index; BP: Blood Pressure; CDS: Chinese Diabetes Society; CVD: Cardiovascular Disease; HbA1c: Glycosylated Hemoglobin A1c; HR: Heart Rate; IDF: International Diabetes Federation; MS: Metabolic Syndrome; SE: Standard Error; T2DM: Type 2 Diabetes; UA: Uric Acid; WC: Waist Circumstance

\section{Acknowledgements}

We sincerely thank Professor Weiping Teng and Zhongyan Shan from the First Affiliated Hospital of China Medical University and the First Affiliated Hospital of Sun Yat-Sen University, and the staff (Chao Chen, Meimei Liu, Liqun Liu, Sicong Wan, Qiwei Fan, Wenlan Min, Weiwei Gao, Ting Chen, Ying Zhang, Tingmie Tu, Yanmei Yuan, Mengyin Wang, Xue Chen, Yiwen Deng, Shuihong Lei, Yan Xiong, Min Zhou, Yongze Li) that participated in this study and the locals.

\section{Authors' contributions}

Primary data collection for the study was performed by L.W., Y.S, L.H., M.Z. and X.L. The secondary data analyses were designed and performed by L.W. and Y.S. The manuscript was written by L.W. and Y.S. The final version was reviewed by M.Z. and X.L. All authors have read and approved the manuscript.

\section{Funding}

This work was supported by the Research Fund for Public Welfare, the National Health and Family Planning Commission of China [grant number 201402005] and the Clinical Research Fund of Chinese Medical Association [grant number 15010010589].

\section{Availability of data and materials}

The dataset of the current study is available from the corresponding author upon reasonable request.

\section{Ethics approval and consent to participate}

This study was approved by the Ethics Committee of the First Affiliated Hospital of China Medical University. Informed written consent was obtained from all participants before data collection.

\section{Consent for publication}

Not applicable.

\section{Competing interests}

The authors declare that they have no competing interests.

\section{Author details}

${ }^{1}$ Department of Endocrinology and Metabolism, The Second Affiliated Hospital of Nanchang University, No.1, Minde Road, Donghu District, Nanchang 330006, China. 'Department of Endocrinology and Metabolism, The First Affiliated Hospital of Sun Yat-sen University, No. 58, ZhongshanEr Road, Guangzhou 510080, China. ${ }^{3}$ Institute for the Study of Endocrinology and Metabolism Diseases in Jiangxi Province, No.1, Minde Road, Donghu District, Nanchang 330006, China.

Received: 5 June 2019 Accepted: 13 January 2020

Published online: 30 January 2020

\section{References}

1. Isomaa B, Almgren P, Tuomi T, Forsén B, Lahti K, Nissén M, et al. Cardiovascular morbidity and mortality associated with the metabolic syndrome. Diabetes Care. 2001;24(4):683-9.

2. Cheung BMY, Wat NMS, Man YB, Tam S, Thomas GN, Leung GM, et al, Development of diabetes in Chinese with the metabolic syndrome a 6-year prospective study. Diabetes Care. 2007;30(6):1430.

3. Wang J, Li H, Kinnunen L, Hu G, Jarvinen TM, Miettinen ME, et al. How well does the metabolic syndrome defined by five definitions predict incident diabetes and incident coronary heart disease in a Chinese population. Atherosclerosis. 2007;192(1):161

4. Mukai N, Doi Y, Ninomiya T, Hata J, Yonemoto K, Iwase M, et al. Impact of metabolic syndrome compared with impaired fasting glucose on the development of type 2 diabetes in a general Japanese population: the Hisayama study. Diabetes Care. 2009;32(12):2288.

5. Mottillo S, Filion KB, Genest J, Joseph $L$, Pilote $L$, Poirier $P$, et al. The metabolic syndrome and cardiovascular risk : a systematic review and metaanalysis. J Am Coll Cardiol. 2010;56(14):1113.

6. Kaur J. A comprehensive review on metabolic syndrome. Cardiol Res Pract. 2014;2014:943162

7. Guixian T. The prevalence of metabolic syndrome in a 11 provinces cohort in China. Chin J Prev Med. 2002;36(5):298. 
8. Gu D, Reynolds K, Wu X, Chen J, Duan X, Reynolds RF, et al. Prevalence of the metabolic syndrome and overweight among adults in China. Lancet. 2005;365(9468):1398.

9. Zimmet P, Magliano DJ, Matsuzawa Y, Alberti G, Shaw JE. The metabolic syndrome: a global public health problem and a new definition. J Atheroscler Thromb. 2005;12(6):295.

10. Wang W, Niu JQ, He S, Sun J, Wang C, Chen H, et al. Epidemiological investigation of metabolic syndrome and analysis of relevant factors in Northeast China. Int J Cardiol. 2009;137(38):150.

11. Chen B, Yang D, Chen Y, Xu W, Ye B, Ni Z. The prevalence of microalbuminuria and its relationships with the components of metabolic syndrome in the general population of China. Clin Chim Acta. 2010;411(9):705.

12. Lao XQ, Zhang $Y$, Wong MCS, Xu Y J, Xu HF, Nie S, et al. The prevalence of metabolic syndrome and cardiovascular risk factors in adults in southern China. BMC Public Health. 2012;12(1):64.

13. Li SL, Yang Q, Lv SY, Zhang YL, Zhang J. Prevalence of the metabolic syndrome in the Yan-an region of Northwest China. J Int Med Res. 2012;40(2):673.

14. Xi B, He D, Hu Y, Zhou D. Prevalence of metabolic syndrome and its influencing factors among the Chinese adults: the China health and nutrition survey in 2009. Prev Med. 2013;57(6):867.

15. Lan Y, Mai Z, Zhou S, Liu Y, Li S, Zhao Z, et al. Prevalence of metabolic syndrome in China: An up-dated cross-sectional study. PLoS One. 2018; 13(4):e0196012.

16. Yi Z, Jing J, Xiuying L, Hongxia X, Jianjun Y, Yuhong Z. Prevalence of the metabolic syndrome among rural original adults in NingXia, China. BMC Public Health. 2010:10(1):140.

17. Wang GR, Li L, Pan YH, Tian GD, Lin WL, Li Z, et al. Prevalence of metabolic syndrome among urban community residents in China. BMC Public Health. 2013;13(1):599.

18. Enkh-Oyun T, Kotani K, Davaalkham D, Davaa G, Ganchimeg U, Angarmurun $D$, et al. Epidemiologic Features of Metabolic Syndrome in a General Mongolian Population. Metab Syndr Relat Disord. 2015;13(4):179.

19. Zhan Y, Yu J, Chen R, Gao J, Ding R, Fu Y, et al. Socioeconomic status and metabolic syndrome in the general population of China: a cross-sectional study. BMC Public Health. 2012;12(1):921.

20. So-Jung P, Hee-Taik K, Chung-Mo N, Byoung-Jin P, Linton John A, Yong-Jae $L$. Sex differences in the relationship between socioeconomic status and metabolic syndrome: The Korean National Health and Nutrition Examination Survey. Diabetes Res Clin Pract. 2012;96(3):400-6.

21. Ferguson TS, Tulloch-Reid MK, Younger NOM, Knight-Madden JM, SammsVaughan M, Ashley D, et al. Prevalence of the metabolic syndrome and its components in relation to socioeconomic status among Jamaican young adults: a cross-sectional study. BMC Public Health. 2010;10(1):307.

22. Cheng L, Yan W, Zhu L, Chen Y, Liu J, Xu Y, et al. Comparative analysis of IDF, ATPIII and CDS in the diagnosis of metabolic syndrome among adult inhabitants in Jiangxi Province, China. PLoS One. 2017:12(12):e0189046.

23. Rampal S, Mahadeva S, Guallar E, Bulgiba A, Mohamed R, Rahmat R, et al. Ethnic Differences in the Prevalence of Metabolic Syndrome: Results from a Multi-Ethnic Population-Based Survey in Malaysia. PLoS One. 2012;7(9):e46365.

24. Moreira GC, Cipullo JP, Ciorlia LADS, Cesarino CB, Vilelamartin JF. Prevalence of metabolic syndrome: association with risk factors and cardiovascular complications in an urban population. PLoS One. 2014;9(9):e105056.

25. Lei C. Prevalence of metabolic syndrome among Shanghai adults in China. Chin J Cardiol. 2003;31(12):909.

26. Li Y, Zhao L, Yu D, Wang Z, Ding G. Metabolic syndrome prevalence and its risk factors among adults in China: A nationally representative crosssectional study. PLoS One. 2018;13(6):e0199293.

27. Das M, Pal S, Ghosh A. Association of metabolic syndrome with obesity measures, metabolic profiles, and intake of dietary fatty acids in people of Asian Indian origin. J Cardiovasc Dis Res. 2010;1(3):130.

28. Torrens Jl, Suttontyrrell K, Zhao X, Matthews KA, Brockwell S, Sowers M, et al. Relative androgen excess during the menopausal transition predicts incident metabolic syndrome in midlife women : study of Women's health across the nation. Menopause. 2009;16(2):257.

29. Monami M, Marchionni N, Masotti G, Mannucci E. IDF and ATP-III definitions of metabolic syndrome in the prediction of all-cause mortality in type 2 diabetic patients. Diabetes Obes Metabol. 2007;9(3):350.

\section{Publisher's Note}

Springer Nature remains neutral with regard to jurisdictional claims in published maps and institutional affiliations.

Ready to submit your research? Choose BMC and benefit from:

- fast, convenient online submission

- thorough peer review by experienced researchers in your field

- rapid publication on acceptance

- support for research data, including large and complex data types

- gold Open Access which fosters wider collaboration and increased citations

- maximum visibility for your research: over $100 \mathrm{M}$ website views per year

At $\mathrm{BMC}$, research is always in progress.

Learn more biomedcentral.com/submissions 